\title{
Features of generalized dynamics of quasiparticles in the presence of an external potential field. Part 2. Minkowski equations and degeneration of mobility in the strong fields
}

\author{
A.D.Suprun, L.V.Shmeleva \\ Faculty of Physics, Kyiv National Taras Shevchenko University, prosp. \\ Glushkova, 4, Kyiv, Ukraine
}

Received January 4, 2016

This article analyses the influence of the external electrostatic field (having an arbitrary space configuration) upon the properties of a free quasi-particle (for example, on the properties of the electron, which was injected into a semiconductor or into a dielectric). Approximations were formulated, that ensure fulfilment of the generalized Minkowski equations for the components of the external field for both methods of description: a quantum method of description within a local frame of reference and a classic method of description within a global frame of reference. It was demonstrated that the generalized dynamics of a quasiparticle in the external potential field might be one of the possible reasons of the abnormal mobility of carriers in the strong fields.

Keywords: external electrostatic field, quasi-particle, Minkowski equations.

Рассматривается влияние внешнего электростатического поля произвольной пространственной конфигурации на свойства свободной квазичастицы типа электрона, инжектированного в полупроводник или диэлектрик. Сформулированы приближения, которые обеспечивают выполнение обобщенных уравнений Минковского между компонентами внешнего поля для обоих способов описания: квантового - в локальной системе отсчета и классического - в глобальной. Показано, что одной из возможных причин экспериментально наблюдаемой аномальной подвижности носителей в сильных полях может быть исследуемая здесь обобщенная динамика квазичастиц во внешнем потенциальном поле.

Про особливості узагальненої динаміки квазічастинки при наявності зовнішнього потенційного поля. Частина 2. Рівняння Мінковського і виродження рухливості при великих полях. А.Д. Супрун, Л.В. Шлельова.

Розглядається вплив зовнішнього електростатичного поля довільної просторової конфігурації на властивості вільної квазічастинки типу електрона, інжектованого у напівпровідник або діелектрик. Сформульовано наближення, які забезпечують виконання узагальнених рівнянь Мінковського між компонентами зовнішнього поля для обох способів опису: квантового - у локальній системі відліку і класичного - у глобальній. Показано, що однією з можливих причин аномальної рухливості носіїв, у сильних полях, що спостерігається експериментально, може бути узагальнена динаміка квазічастинок, що досліджується, у зовнішньому потенційному полі. 


\section{Introduction}

Features of the dynamics of a free quasi-particle have been analyzed in [1] through the example of the electron, which was injected into a semiconductor. It has been shown that these features are based on the one of the main characteristics of the excited states of the condensed matters, that is, they are based on the dispersive dependence of energy (or dispersive dependence of frequency) on the wave vector $[2-5]$. These articles analyse the electron within the conduction band provided that this electron is in the external electrostatic field, which has an arbitrary and a weakly variable configuration in space. The Minkowski equations for the components of the external field were derived for both methods of description of the dynamics of a quasiparticle, i.e. for the quantum and classic methods of description. It was demonstrated that the observed [6,7] abnormal mobility of carriers in the strong fields may include a dynamic factor as one of the mechanisms, which may explain such an anomaly.

\section{Materials and Methods. Fundamental Relationships}

Certain features of the dynamics of the electron, which was injected into a semiconductor, are analyzed, first, based on the dimensionless equations of motion:

$$
\dot{\mathbf{r}_{0}}=\beta+\sigma_{\mathbf{p}} ; \quad \dot{\mathbf{p}}=\mathbf{G}+\delta \mathbf{G},
$$

which were discussed in detail in the first part of the article [8] (see equations (45) of this part). Here $\beta$ denotes the dimensionless velocity of the quasiparticle with the components $\beta_{\alpha}: \beta=\mathbf{e}_{\alpha} \beta_{\alpha}$, where $\beta_{\alpha} \equiv \sin \left(p_{\alpha}\right)$. G denotes the "global" field, which is eventually connected with a given external field. Vectors $\sigma_{\mathbf{p}}$ and $\delta \mathbf{G}$ in the components have the form as follows [8] (in the first part - this is the equations (46) and right after it):

$$
\begin{gathered}
\sigma_{\mathbf{p}}^{\alpha} \equiv\left[\left(\hat{\mu}^{-1} \mathbf{F} \cdot \frac{\partial \xi}{\partial p_{\alpha}}\right)+\left(\mathbf{\Phi} \cdot \frac{\partial \mathbf{r}_{*}}{\partial p_{\alpha}}\right)-\left(\left(\mathbf{r}_{0}-\xi\right) \cdot \frac{\partial}{\partial p_{\alpha}}\left(\hat{\mu}^{-1} \mathbf{F}\right)\right)\right] \\
\delta G_{\alpha} \equiv\left\{\frac{F_{\alpha}}{\mu_{\alpha}}-G_{\alpha}-\left[\left(\hat{\mu}^{-1} \mathbf{F} \cdot \frac{\partial \xi}{\partial x_{0}^{\alpha}}\right)+\left(\mathbf{\Phi} \cdot \frac{\partial \mathbf{r}_{*}}{\partial x_{0}^{\alpha}}\right)-\left(\left(\mathbf{r}_{0}-\xi\right) \cdot \frac{\partial}{\partial x_{0}^{\alpha}}\left(\hat{\mu}^{-1} \mathbf{F}\right)\right)\right]\right\} .
\end{gathered}
$$

Here $\mathbf{F}$ denotes the "local" force, $\widehat{\mu}$ denotes the effective mass tensor, $\mathbf{r}_{*}$ denotes point of conditional localization of the field source, $\xi$ denotes a some coordinate, $\mathbf{r}_{0}$ denotes the vector, which has the following components $x_{0}^{\alpha}(\alpha=1,2,3), \Phi_{\alpha} \equiv\left(\mathbf{r}_{*} \cdot \frac{\partial \boldsymbol{\Pi}_{\mathbf{r}_{*}}}{\partial x_{*}^{\alpha}}\right), \boldsymbol{\Pi}_{\mathbf{r}_{*}}$ denotes a dimensionless representation of a given force. The dimensionless forces $\mathbf{G}$ and $\mathbf{F}$ along with the dimensionless vector parameters $\xi$ and $\mathbf{r}_{*}$ are not specified. They are determined by four Hamiltonian conditions of the dynamics of a quasiparticle.

It was shown in [8] (see equality (47)) that condition $\sigma_{\mathbf{p}}=0$ is the main one among the other conditions. As it follows from the equation (2), this main condition has the form of three differential equations for the canonically conjugate variables, namely, pulse $\mathbf{p}$ and coordinate of the conditional localization of a quasiparticle $\mathbf{r}_{0}$ :

$$
\left[\left(\widehat{\mu}^{-1} \mathbf{F} \cdot \frac{\partial \xi}{\partial p_{\alpha}}\right)+\left(\boldsymbol{\Phi} \cdot \frac{\partial \mathbf{r}_{*}}{\partial p_{\alpha}}\right)-\left(\left(\mathbf{r}_{0}-\xi\right) \cdot \frac{\partial}{\partial p_{\alpha}}\left(\widehat{\mu}^{-1} \mathbf{F}\right)\right)\right]=0, \quad \alpha=1,2,3 .
$$

Another such condition $\delta \mathbf{G}=\mathbf{0}$, which (as it follows from the equation (3)) also has the form of three differential equations in respect to the canonically conjugate variables $\mathbf{r}_{0}$ and $\mathbf{p}$ has the form:

$$
\frac{F_{\alpha}}{\mu_{\alpha}}-G_{\alpha}=\left(\widehat{\mu}^{-1} \mathbf{F} \cdot \frac{\partial \xi}{\partial x_{0}^{\alpha}}\right)+\left(\boldsymbol{\Phi} \cdot \frac{\partial \mathbf{r}_{*}}{\partial x_{0}^{\alpha}}\right)-\left(\left(\mathbf{r}_{0}-\xi\right) \cdot \frac{\partial}{\partial x_{0}^{\alpha}}\left(\widehat{\mu}^{-1} \mathbf{F}\right)\right), \quad \alpha=1,2,3 .
$$

These two conditions ( $\sigma_{\mathbf{p}}=0$ and $\delta \mathbf{G}=\mathbf{0}$ ) bring the equations (1) to the following form:

$$
\dot{\mathbf{r}_{0}}=\beta ; \quad \dot{\mathbf{p}}=\mathbf{G},
$$


and determine the dynamics of the point of conditional localization of the quasiparticle $\mathbf{r}_{0}$ in respect to the "global" frame of reference, which is connected with the crystal. The first (the left) of the equations (6) are similar to the analogous equations of a free quasiparticle and all procedure of getting the equations (1) in the form (6) may be regarded as a generalization for the case of dynamics in the presence of an external field.

In order to ensure the Hamiltonian nature of the dynamics of a quasiparticle, the following scalar equation $h=\Omega-\left\{\mathbf{r}_{0}-\xi\right\} \cdot \mathbf{G}+\mathbf{p} \cdot \dot{\xi}$ was considered (in [8] this equation is given immediately after the formula (41)). This equation ties the dimensionless Hamiltonian $h$ of the system "injected electron - external field" with the quantum eigenvalue $\Omega$ of this system. This definition includes components of three arbitrary vectors: $\mathbf{G}, \xi$, and $\mathbf{r}_{*}$ (the latter of them is only included to the $\Omega$ ). Availability of these vectors makes it possible to raise the question concerning coincidence of the Hamiltonian $h$ and the eigenvalue $\Omega$. It is very important from the physical standpoint, because of, on the one hand, the Hamiltonian of any closed system is constant in time, while, on the other hand, eigenvalue $\Omega$ must be a constant in time as well. The condition $h=\Omega$ results in the scalar condition in the form of the ordinary differential equation in respect of time $\tau$ :

$$
\mathbf{p} \cdot \dot{\xi}=\left\{\mathbf{r}_{0}-\xi\right\} \cdot \mathbf{G}
$$

In [8] considered another condition relating the force components $\mathbf{F}$ and $\mathbf{G}$. They are the same force, but in the different frames of reference, and are connected with each other by the following vector condition: $\boldsymbol{\Pi}_{\mathbf{r}_{*}}-\mathbf{G}=\widehat{\mu}^{-1} \mathbf{F}$ (see equations (31) or (50) in [8]). This vector condition decomposed into three separate conditions (in the same manner as conditions (4) and (5)):

$$
\frac{F_{\alpha}}{\mu_{\alpha}}+G_{\alpha}=\Pi_{\mathbf{r}_{*}}^{\alpha}, \quad \alpha=1,2,3 .
$$

That is, actually there exist 10 conditions for 12 components of four vectors. Such an overdetermination ensures some freedom of choice. For example, it is possible to consider that one of these vectors is the single-component vector. It is most likely that the vector $\boldsymbol{\Pi}_{\mathbf{r}_{*}}$, which is the predetermined function of $\mathbf{r}_{*}$, may be treated as such single-component vector.

\section{Results and Discussion. Some Applications}

3.1. Minkowski Equations: Relations between the "global" field G and the "local" field F. In order to determine relations between the "global" field $\mathbf{G}$ and the "local" field $\mathbf{F}$, it is necessary to utilise equations (5) and (8), but in the vector representations:

$$
\begin{gathered}
\widehat{\mu}^{-1} \mathbf{F}+\mathbf{G}=\boldsymbol{\Pi}_{\mathbf{r}_{*}} ; \\
\widehat{\mu}^{-1} \mathbf{F}-\mathbf{G}=\sigma_{\mathbf{r}_{0}} .
\end{gathered}
$$

Here $\sigma_{\mathbf{r}_{0}}$ denotes the vector, which was determined by analogous to the vector $\sigma_{\mathbf{p}}$ (see definition (2)):

$$
\sigma_{\mathbf{r}_{0}}^{\alpha} \equiv\left[\left(\widehat{\mu}^{-1} \mathbf{F} \cdot \frac{\partial \xi}{\partial x_{0}^{\alpha}}\right)+\left(\boldsymbol{\Phi} \cdot \frac{\partial \mathbf{r}_{*}}{\partial x_{0}^{\alpha}}\right)-\left(\left(\mathbf{r}_{0}-\xi\right) \cdot \frac{\partial}{\partial x_{0}^{\alpha}}\left(\widehat{\mu}^{-1} \mathbf{F}\right)\right)\right] .
$$

In respect of the vector $\mathbf{G}$, system of equations (9), (10) is the system of algebraic equations, and this fact makes it possible to find out this force:

$$
\mathbf{G}=\frac{1}{2}\left(\boldsymbol{\Pi}_{\mathbf{r}_{*}}-\sigma_{\mathbf{r}_{0}}\right)
$$

From the formal standpoint, with the help of the system of equations (9), (10) it is possible to find out the force $\mathbf{F}$ as well: 


$$
\mathbf{F}=\frac{1}{2} \widehat{\mu}\left(\boldsymbol{\Pi}_{\mathbf{r}_{*}}+\sigma_{\mathbf{r}_{0}}\right)
$$

However, it is evident from the definition (11), that the equality (13) is the subsystem of the differential equations, which complements the subsystem (4) and equation (7) up to the complete system in respect of the vectors: $\mathbf{r}_{*}, \xi$, and $\mathbf{F}$. If there were no amendments $\sigma_{\mathbf{r}_{0}}$ in the equations (12) and (13), then Minkowski equations [9, 10] would be valid: $F_{\alpha}=G_{\alpha} / \sqrt{1-\beta_{\alpha}^{2}}$, which were obtained by exclusion of the field $\boldsymbol{\Pi}_{\mathbf{r}_{*}}$ from the equations (12) and (13). Here definitions of the components of the velocity vector $\beta_{\alpha} \equiv \sin \left(p_{\alpha}\right)$, as well as definitions of the elements of the diagonal tensor of the effective mass $\mu_{\alpha} \equiv 1 / \cos \left(p_{\alpha}\right)$, were also taken into consideration. From these two definitions follows that $\mu_{\alpha}=1 / \sqrt{1-\beta_{\alpha}^{2}}$. Minkowski equations determine interconnection between the force $\mathbf{F}$, which is only included to the Schrödinger equation [8] (see equations (34) in [8]):

$$
\frac{1}{2} \cdot \frac{\partial^{2} \phi_{\alpha}}{\partial \rho_{\alpha}^{2}}+\left(\rho_{\alpha}+\xi_{\alpha}\right) F_{\alpha} \phi_{\alpha}=0 ; \quad \alpha=1,2,3,
$$

and the "global" force $\mathbf{G}$. The force $\mathbf{F}$ is formulated in its own frame of reference in respect of the point $\mathbf{r}_{0}$. Variables $\rho_{\alpha}$ denote components of the vector $\rho \equiv \mathbf{r}-\mathbf{r}_{0}$ [8]. Presence of the correction $\sigma_{\mathbf{r}_{0}}^{\alpha}$ modifies the Minkowski equations to the form: $F_{\alpha}=\left(G_{\alpha}+\sigma_{\mathbf{r}_{0}}^{\alpha}\right) / \sqrt{1-\beta_{\alpha}^{2}}$.

\subsection{About abnormal motility of quasiparticles in an external potential field.}

Here will be considered application of the obtained results in respect to one of possible interpretations of the abnormal mobility [6,7] of the charged carrier in crystal in the external electrostatic field.

If solutions of the system of equations (4), (7), (9), and (10) (solution of (10) must be found taking into account the equation (11)) for all four unknown vectors: $\mathbf{F}, \mathbf{G}, \xi$, and $\mathbf{r}_{*}$ would be found, then these vectors in the general case must be functions of time $\tau$, pulse $\mathbf{p}$, and coordinate $\mathbf{r}_{0}$. In the conservative case, when there is no any explicit dependence on time in these equations, all quantities will only be functions of $\mathbf{p}$ and $\mathbf{r}_{0}$, for example: $\mathbf{G}=\mathbf{G}\left(\mathbf{p}, \mathbf{r}_{0}\right)$. Farther this case will be considered as physically the most typical. In the simplest case, this dependence may be represented in the form as follows:

$$
\mathbf{G}\left(\mathbf{p}, \mathbf{r}_{0}\right)=\mathbf{G}_{0} \pm \tau_{d y n}^{-1} \mathbf{p} \pm k_{d y n} \mathbf{r}_{0}
$$

Here $\tau_{d y n}$ denotes the dimensionless relaxation time, value of which is determined in accordance with the explicit form of the solution of the system of equations (4), (7), (9), and (10). Origination of $k_{d y n}$ parameter is the same, but this parameter has its specific physical significance as an elastic dimensionless constant. Without the direct solution of the system $(4),(7),(9),(10)$ it is impossible to determine what should be signs in the expansion (14).

The second and the third summands in this series may also take into account other physical processes, except for those dynamic processes, which are analyzed within this article. These processes have not been considered, since aim was to focus on "clean" dynamics of the electron (injected into the crystal) in an external field. Therefore, in order to ensure the adequate analysis of the abnormal mobility [6, 7], other factors must be additionally taken into consideration, for example, effects of deceleration of the electron due to electron-phonon interaction. These factors were analyzed in details in [11]. In particular it has been shown that such deceleration has the form: " $-\tau_{e p h}^{-1} \mathbf{p}$ ", where $\tau_{e p h}$ denotes the dimensionless time of the electron-phonon relaxation. What is more, the dimensional analogue of loss factor " $\tau_{\text {eph }}^{-1} \mathbf{p}$ " near the threshold is estimated at [11] as the value of the order of $0.025 \mathrm{eV} / \AA$. It has been also noted in [11], that depending on the physical mechanisms of the relaxation, one of which was analyzed in [7], the losses in the course of the electron deceleration may achieve $0.06 \mathrm{eV} / \AA$, but cannot be lesser than $0.01 \mathrm{eV} / \AA$. In this respect, the second summand in the series (14) will always have the form: " $-\tau_{r e l}^{-1} \mathbf{p}$ ", where

$$
\tau_{r e l}^{-1}=\tau_{e p h}^{-1} \pm \tau_{d y n}^{-1}
$$

Therefore, $\tau_{d y n}$ factor may only influence upon the value of the electron deceleration within the limits from 0.01 to $0.06 \mathrm{eV} / \AA$, and this fact makes it possible to estimate the value of the electron deceleration. 
Taking into account that the dimensional analogue of the difference $\tau_{e p h}^{-1} \mathbf{p}-\tau_{d y n}^{-1} \mathbf{p}$ near the threshold cannot be lesser than $0.01 \mathrm{eV} / \AA$, as well as taking into account the fact that the dimensional analogue of $\tau_{e p h}^{-1} \mathbf{p}$ factor is equal to $0.025 \mathrm{eV} / \AA$, it is possible to make estimation of the dimensional analogue of $\tau_{d y n}^{-1}\left|\mathbf{p}_{t h r}\right|$ factor. Its value cannot exceed $0.015 \mathrm{eV} / \AA$. Here $\left|\mathbf{p}_{t h r}\right|$ denotes the modulus of the threshold value of the dimensionless wave pulse.

The presented values make it possible, first, to estimate the value of $\tau_{e p h}$. For this let us denote the dimensional value of the electron-phonon relaxation as $0.025 \mathrm{eV} / \AA$. Then, such relationship is valid:

$$
t_{e p h}^{-1}\left|\mathbf{P}_{t h r}\right|=L_{e p h}
$$

Here $t_{e p h}$ denotes the real time of the electron-phonon relaxation, while $\left|\mathbf{P}_{t h r}\right|$ denotes the modulus of the threshold value of the real wave momentum. It is possible to represent condition (16) in the dimensionless form with the help of the relationships between the real and dimensionless values: $\left|\mathbf{P}_{t h r}\right|=m_{*} C_{*}\left|\mathbf{p}_{t h r}\right|$,

$$
t_{e p h}^{-1}=\left(\left|M_{b_{0}}\right| / \hbar\right) \tau_{e p h}^{-1}
$$

These relationships have been discussed in details in [1]. Here $m_{*} \equiv \hbar^{2} /\left(\left|M_{b_{0}}\right| b_{0}^{2}\right)$ denotes the effective mass of the carrier in the conduction band (in [1] this value was denoted as $m_{f}$ ); $C_{*} \equiv b_{0}\left|M_{b_{0}}\right| / \hbar$ denotes the maximum (threshold) velocity of this carrier for this band (in [1] this value was denoted as $C_{f}$ ). Taking into account this fact in the equation (16), as well as assuming that $\left|\mathbf{p}_{t h r}\right|=\pi / 2$, it is possible, at last, to obtain: $\tau_{e p h}^{-1}=2 b_{0} L_{e p h} / \pi\left|M_{b_{0}}\right|$. For estimations, we will use the following values: $b_{0}=1 \AA$, as well as $\left|M_{b_{0}}\right|=1 \mathrm{eV}$. Then: $\tau_{e p h}^{-1}=0.0159$, and, respectively, $\tau_{e p h}=62.83$. Taking into account $(17)$, time of real electron-phonon deceleration can be found as well: $t_{\text {eph }} \simeq 4 \cdot 10^{-14} \mathrm{c}$. And taking into account the interrelation between $\tau_{d y n}$ and $\tau_{e p h}$, which was already discussed above, it is possible to obtain similar estimates for $\tau_{d y n}$ and $t_{d y n}: \tau_{d y n}=104.72, t_{d y n} \approx 7 \cdot 10^{-14} \mathrm{c}$. And, at last, the range of values $\tau_{\text {rel }}^{-1}$, based on the definition (15), will be determined as follows:

$$
\tau_{\text {rel }}^{-1}=0.0159 \pm 0.0096=0.0063 \div 0.0255 \text {. }
$$

Such a detailed analysis of the range of values of $\tau_{r e l}^{-1}$ is required in order to estimate the abnormal mobility.

For $k_{d y n}$ parameter, similar estimates may be made as well. For example, it is possible to make the estimates that are connected with the curvature of the conduction band at the interface surface with other materials. Such curvature is described with the help of the factor, which is similar to the third summand in the equation (14), but this factor may also have two signs (depending on the interface material). For the simplicity of further analysis of the abnormal mobility, we will neglect by this summand and will assume that, for example, curvature of the band largely compensates the third summand in the equation (14). Then, equation (14) will take the following form: $\mathbf{G}\left(\mathbf{p}, \mathbf{r}_{0}\right)=\mathbf{G}_{0}-\mathbf{p} / \tau_{\text {rel }}$. Therefore, the second (the right) equation in the expression (6) will take the following form: $\dot{\mathbf{p}}=\mathbf{G}_{0}-\tau_{\text {rel }}^{-1} \mathbf{p}$. In accordance with these approximations, it is possible based on the condition $\dot{\mathbf{p}}=\mathbf{0}$ to find the stationary solution of this equation as follows: $\mathbf{p}_{\text {stc }}=\tau_{r e l} \mathbf{G}_{0}$, where value of the stationary wave pulse is proportional to the external field $\mathbf{G}_{0}$.

At last, mobility can be analyzed using the ratio for the velocity components: $\beta_{\alpha} \equiv \sin \left(p_{\alpha}\right)$, which definitions are given after the equations of motion (1). In result of the substitution of components of the vector $\mathbf{p}_{\text {stc }}=\tau_{\text {rel }} \mathbf{G}_{0}$ to this equality, it is possible to obtain the following expression for the stationary velocity:

$$
\beta_{s t c}^{\alpha}=\sin \left(\tau_{r e l} G_{0}^{\alpha}\right) .
$$

This dependence ensures the exact coincidence with the description of the similar dependence for the charged carrier in the molecular-based doped polymer [6]. Namely, first increases with increasing field, and after reaching a maximum, when $G_{0, \text { max }}^{\alpha}=\pi \tau_{\text {rel }}^{-1} / 2$, begins to decrease. As concerns the range of values of 
$\tau_{r e l}^{-1}$, which is presented in (18), it is possible to obtain that: $G_{0, \max }^{\alpha}=0.01 \div 0.04$. Because the dimensionless value of this force is determined similarly to the dimensionless force $\boldsymbol{\Pi}_{\mathbf{r}_{*}} \equiv-b_{0} \mathbf{J}_{\mathbf{R}_{*}} /\left|M_{b_{0}}\right|$ (in the first part [8] this dimensionless force determined immediately after the formula (12)), its dimensional value $\widetilde{G}_{0, \max }^{\alpha}$ must be determined as follows: $\widetilde{G}_{0, \max }^{\alpha}=\left|M_{b_{0}}\right| G_{0, \max }^{\alpha} / b_{0}$. Then, the above-presented numerical values: $b_{0}=1 \AA$ and $\left|M_{b_{0}}\right|=1 \mathrm{eV}$, will determine this force as follows: $\widetilde{G}_{0, \max }^{\alpha}=(1 \div 4) \cdot 10^{8} \mathrm{eV} / \mathrm{m}$. For relevant strength of the field, we will have the following value: $\widetilde{G}_{0, \max }^{\alpha} / e=(1 \div 4) \cdot 10^{8} \mathrm{~V} / \mathrm{m}$. This value is lesser than the value of the electrostatic threshold of the material for a perfect crystal by $2 \div 3$ orders. In non-ideal crystals, the value of electrostatic threshold of material may be smaller, i.e. closer to the obtained values: $\widetilde{G}_{0, \max }^{\alpha} / e=(1 \div 4) \cdot 10^{8} \mathrm{~V} / \mathrm{m}$. In the actual practice, values of the lattice constant may be substantially higher than $1 \AA$, while width of the conduction band may be substantially lower than 1 $\mathrm{eV}$. This may result in the fact that strength of the field may be lesser than the obtained value by $1 \div 2$ orders.

In conclusion, we will present results of the numerical analysis for the differential coefficient of mobility (derivative of the velocity with respect to the field strength). For this, it is necessary to make a partial transition from the dimensionless values in the definition of the stationary velocity (19) to the dimensional its values. In addition to using the above represented definition of force $G_{0, \max }^{\alpha}=b_{0} \widetilde{G}_{0, \max }^{\alpha} /\left|M_{b_{0}}\right|$, is used also the definition of the dimensionless velocity $\beta_{s t c}^{\alpha} \equiv V_{s t c}^{\alpha} / C_{*}$, defined in [1]. As concerns the relaxation time $\tau_{\text {rel }}$, it would be more conveniently to use the dimensionless representation, which was determined numerically with the help of the equation (18). At the same time, it is also necessary to use the above-presented explicit value of the maximum velocity $C_{*} \equiv b_{0}\left|M_{b_{0}}\right| / \hbar$. As the result, it is possible to obtain the following expression for the differential coefficient of mobility: $\widetilde{\mu}_{s t c}^{\alpha}=\widetilde{\mu}_{s t c}^{0, \alpha} \cos \left(\tau_{r e l} G_{0}^{\alpha}\right)$, where $\widetilde{\mu}_{\text {stc }}^{0, \alpha} \equiv b_{0}^{2} \tau_{r e l} e / \hbar$ denotes the peak value of the differential coefficient of mobility. For the used here value of the lattice constant $b_{0}=1 \AA$, one can get an estimate: $\widetilde{\mu}_{\text {stc }}^{0, \alpha}=\left(1.6 \cdot 10^{-5} / \tau_{r e l}^{-1}\right)\left[\mathrm{m}^{2} / \mathrm{Vs}\right] \equiv\left(0.16 / \tau_{r e l}^{-1}\right)\left[\mathrm{cm}^{2} / \mathrm{Vs}\right]$. As concerns the values $\tau_{r e l}^{-1}$ within the range of values in accordance with the equation (18), it is possible to obtain the following maximum value for the coefficient of mobility: $\widetilde{\mu}_{s t c}^{0, \alpha}=25.4\left[\mathrm{~cm}^{2} / \mathrm{Vs}\right]$. In the weak fields: $\left(\left|\tau_{\text {rel }} G_{0}^{\alpha}\right|<<1\right)$, coefficient of mobility $\widetilde{\mu}_{s t c}^{\alpha}$ will have exactly this value. At the same time, in the strong fields, which are determined by the edges of the Brillouin zone: $\left|\tau_{r e l} G_{0}^{\alpha}\right| \sim \pi$, this coefficient has the abnormal sign: $\widetilde{\mu}_{s t c}^{\alpha}=-25.4\left[\mathrm{~cm}^{2} / \mathrm{Vs}\right]$. In [7] was noted that under certain conditions, value of the negative coefficient of mobility may achieve the level of several hundreds of $\mathrm{cm}^{2} / \mathrm{V} \cdot \mathrm{s}$. It follows from the definition $\widetilde{\mu}_{s t c}^{0, \alpha} \equiv b_{0}^{2} \tau_{\text {rel }} e / \hbar$ that in the case of application of more actual values of the lattice constant (as compared with the assumed value at the level of $b_{0}=1 \AA$ ), it is possible to obtain the greater values of the mobility coefficients. For example, if $b_{0}=2 \AA$, then: $\widetilde{\mu}_{s t c}^{\alpha}=-101.6\left[\mathrm{~cm}^{2} / \mathrm{Vs}\right]$.

\section{Conclusions}

The dynamic properties of the quasiparticles (of type of the electrons, which are injected into a semiconductor or dielectric) in the external electrostatic field of the general space configuration, were analyzed.

Relevant relationships were derived in respect of the components of the external field for the quantum method of description (local frame of reference), on the one hand, and for the classic method of description (global frame of reference), on the other hand. These relationships are formally coincide with the generalized Minkowski equations. The analysis of the experimental data in respect of the abnormal mobility of carriers in the strong fields $[6,7]$ has demonstrated that one of the reason of this anomaly may be connected with the investigated here generalized dynamics of the quasiparticles in the external potential field. Relevant estimates were made in order to determine the values of the field, at which the differential coefficient of mobility (derivative from the velocity by the field strength) degenerates and assumes zero value. Calculated values of the field are lesser than the values of the electrostatic threshold of the material by $2 \div 3$ orders (while sometimes - more than by $2 \div 3$ orders) [7]. Estimates of the abnormal (negative) mobility, fulfilled in accordance with the results of the theoretical calculations, which were obtained here, have the order of magnitude, close to that the obtained experimentally [7]. 


\section{References}

1. A. D. Suprun, L. V. Shmeleva, Functional Materials, 19, 508 (2012).

2. M. F. Smith, M. B. Walker, Phys. Rev. B, 67, 214509 (2003).

3. S. D. Sarma, S. Adam, E. H. Hwang et al., Rev. Mod. Phys., 83, 407 (2011).

4. O. P. Verkhoglyadova, B. T. Tsurutani, G. S. Lakhina, J. Geophys. Res.: Space Phys., 118, 7695 (2013).

5. A. V. Filippov, A. G. Zagorodny, A. I. Momot et al., Zh. Eksp. Teor. Fiz., 108, 497 (2009).

6. M. Novo, M. van der Auweraer, F. C. de Schryver et al., Phys. Stat. Sol. B., 177, 223 (1993).

7. K. W. Kim, V. V. Korotyeyev, V. A. Kochelap et al., J. Appl. Phys., 96, 6488 (2004).

8. L.V. Shmeleva, A.D. Suprun, Functional Materials, 22, 524 (2015).

9. Yu. P. Terletsky, Yu. P. Rybakov, Electrodynamics. Vysshaya Shcola, Moscow (1990) [in Russian].

10. V.G.Levich, Course of Theoretical Physics. Vol. 1. Part 2, Chapter 2, §12, PhysMathGiz, Moscow (1962) [ir Russian].

11. K. K. Thornber, Richard P. Feynman, Phys. Rev. B., 1, 4099 (1970). 